\title{
A CHARACTERIZATION OF SUZUKI'S SIMPLE GROUPS
}

\author{
SHI WUJIE ${ }^{1}$
}

(Communicated by Warren J. Wong)

\begin{abstract}
In this short paper we have characterized Suzuki's simple groups $S_{z}\left(2^{2 m+1}\right), m \geq 1$ using only the set $\pi_{e}(G)$ of orders of elements in the group $G$. That is, we have

Theorem 2. Let $G$ be a finite group. Then $G \simeq S_{z}\left(2^{2 m+1}\right), m \geq 1$ if and only if $\pi_{e}(G)=\left\{2,4\right.$, all factors of $\left(2^{2 m+1}-1\right),\left(2^{2 m+1}-2^{m+1}+1\right)$, and $\left.\left(2^{2 m+1}+2^{m+1}+1\right)\right\}$.
\end{abstract}

Suzuki's simple groups $S_{z}\left(2^{2 m+1}\right), m \geq 1$ is a family of Zassenhaus groups ( $Z$-groups) of odd degree [9]. In [4] we characterized another family of Zassenhaus groups of odd degree $L_{2}\left(2^{m}\right)$ using only the set of orders of elements in the group $G$. That is, let $\pi_{e}(G)$ denote the set of orders of elements in the group $G$. Then we have proved the following theorem.

Theorem 1. Let $G$ be a finite group. Then $G \simeq L_{2}\left(2^{m}\right), m \geq 2$ if and only if $\pi_{e}(G)=\left\{2\right.$, all factors of $\left(2^{m}-1\right)$ and $\left.\left.2^{m}+1\right)\right\}$.

In this short paper, we continue this work and obtain the following theorem.

Theorem 2. Let $G$ be a finite group. Then $G \simeq S_{z}\left(2^{2 m+1}\right), m \geq 1$ if and only if $\pi_{e}(G)=\left\{2,4\right.$, all factors of $\left(2^{2 m+1}-1\right),\left(2^{2 m+1}-2^{m+1}+1\right)$, and $\left.\left(2^{2 m+1}+2^{m+1}+1\right)\right\}$.

Since the simple $Z$-groups of odd degree consists of $L_{2}\left(2^{m}\right)(m \geq 2)$ and $S_{z}\left(2^{2 m+1}\right),(m \geq 1)$, we have

Corollary. Let $G$ be a finite group and $M$ a simple Z-groups of odd degree. Then $G \simeq M$ if and only if $\pi_{e}(G)=\pi_{e}(M)$.

Before starting the proof we give a remark about the set $\pi_{e}(G)$ in Theorem 2. Since $2^{2 m+1}-1 \not \equiv 0(\bmod 3)$ and $\left(2^{2 m+1}-2^{m+1}+1\right) \cdot\left(2^{2 m+1}+2^{m+1}+1\right)=$ $2^{4 m+2}+1 \not \equiv 0(\bmod 3), 3 \notin \pi_{e}(G)$. And $2^{2 m+1}-1$ is prime to 5 , but $5 \in \pi_{e}(G)$ by $2^{4 m+2}+1 \equiv 0(\bmod 5)$.

Proof of Theorem 2. We need only prove the sufficiency by [3, XI Theorem 3.10].

Received by the editors June 25, 1990 and, in revised form, October 9, 1990.

1980 Mathematics Subject Classification (1985 Revision). Primary 20D05, 20 D06.

${ }^{1}$ The author would like to thank the Department of Pure Mathematics of the University of Sydney for the hospitality. 
Let $G$ be a finite group satisfying the condition of the theorem. Then $G$ is a CIT group [10].

1. $G$ is a nonsolvable CIT group. Let $r$ be a prime divisor of $2^{2 m+1}-1$. Then we have $r \neq 2$ and $r \neq 5$. Suppose that $G$ is solvable. Then $G$ contains a Hall $\{2,5, r\}$-subgroup $C$. The assumptions imply that $C$ is a solvable group in which every element has prime power order. From [2, Theorem 1] we have $|\pi(C)| \leq 2$; a contradiction.

Then $G$ has a normal 2-group $N$ such that $G / N$ is isomorphic to one of the following groups $[10$, III, Theorem 5$]$ :

$L_{2}(q), q=2^{n}, n \geq 2$ or $q=p$ is a Fermat prime or Mersenne prime, or $q=9 ; S_{z}\left(2^{2 n+1}\right), n \geq 1 ; L_{3}(4) ; M_{9}$.

2. $\bar{G}=G / N \simeq S_{z}\left(2^{2 m+1}\right), m \geq 1$. Since $3 \notin \pi_{e}(G), \bar{G}$ can only be $S_{z}\left(2^{2 n+1}\right), n \leq m$. Again by the assumption, the maximal order of elements of $\bar{G}$ is $2^{2 m+1}+2^{m+1}+1$. We infer that $n=m$.

3. $G \simeq S_{z}\left(2^{2 m+1}\right), m \geq 1$. It is enough to prove that $N=1$. If not, we can assume $N \neq 1$ is an elementary abelian 2-group without loss of generality. Considering the Sylow 5-normalizer $N_{\bar{G}}\left(\bar{P}_{5}\right)$ of $\bar{P}_{5}$ in $\bar{G}$, where $\bar{P}_{5} \in \operatorname{Syl}_{5} \bar{G}$, we see that the cyclic subgroup $\mathbb{Z}_{4}$ is contained in $N_{\bar{G}}\left(\bar{P}_{5}\right)$. Then $\bar{G}$ has a subgroup $\bar{U}=\mathbb{Z}_{4}\left[\mathbb{Z}_{5}\right]$. Again considering the inverse image $U$ of $\bar{U}$ in $G$, $|U|=2^{2} \cdot 5 \cdot|N|$ and $U$ has a normal series

$$
U>\mathbb{Z}_{5}[N]>N>1 .
$$

Taking an element $g$ of order 5 in $U$, there is an element $c$ of order 4 in $U$ such that $c$ normalizes $\langle g\rangle$, i.e. $c^{-1} g c=g^{2}$. Set $H=\langle g, c\rangle$. Then $H=\mathbb{Z}_{4}\left[\mathbb{Z}_{5}\right]$ and $U=H N$. Since the elementary abelian 2-group $N$ admits the involutive automorphism induced by the element $t=c^{2}$, we have $\left|C_{N}(t)\right|^{2} \geq$ $|N|$. Suppose $\left|C_{N}(t)\right|^{2}>|N|$. As every squares $t_{1}$ and $t_{2}$ of elements of order 4 in $H$ are conjugate, we consider the centralizer $C_{N}\left(t_{1}\right)$ and $C_{N}\left(t_{2}\right)$ of $t_{1}$ and $t_{2}$ in $N$, where $t_{1} \neq t_{2}$. Then $C_{N}\left(t_{1} t_{2}\right)=C_{N}\left(t_{1}\right) \cap C_{N}\left(t_{2}\right) \neq 1$ for every such $t_{1}, t_{2}$. But $\left|t_{1} t_{2}\right|=5, G$ contains element of order 10; a contradiction. And if $\left|C_{N}(t)\right|^{2}=|N|$, we have $C_{N}(t)=[N, t]$ by $\left(n \cdot n^{t}\right)^{t}=n \cdot n^{t}, \forall n \in N$. In this case we prove that $U$ has the elements of order 8. If not, then $G^{4}=1$. Put $x \in N$ and $[x, c] \neq 1$, we have

$$
(x c)^{2}=x^{2} x^{-1} c x c^{-1} c^{2}=\left[x, c^{-1}\right] t
$$

and

$$
(x c)^{4}=\left[\left[x, c^{-1}\right], t\right]=1 .
$$

So $[N, c, t]=1$ and $[N, c] \leq C_{N}(t)=[N, t]$. Hence $[N, c, c] \leq[N, t, c]=$ $[N, c, t]=1$ by the three subgroup lemma. Also since $\left[N, c^{2}\right]=[N, c][N, c]$ - $[N, c, c]=1$, which means that $t=c^{2}$ centralizes $N$, it is contrary to $\left|C_{N}(t)\right|^{2}=|N|$. Therefore $N=1$ and $G \simeq S_{z}\left(2^{2 m+1}\right), m \geq 1$. The theorem is proved.

Remark 1. In [5, Theorem 3] we have characterized $S_{z}\left(2^{2 m+1}\right)$ using the conditions of the order of a group $G$ and the set of orders of elements in $G$. Theorem 2 is clearly a generalization of the above-mentioned conclusion. 
Remark 2. The condition of odd degree cannot be removed. In fact, we cannot characterize $L_{2}(9)$, a simple $Z$-group of even degree, using only the condition of $\pi_{e}(G)$.

Remark 3. Except $L_{2}(9) \simeq A_{6}$, we have characterized all simple groups in which every element has prime power order [11, Theorem 16].

That is, we have the following conclusion:

Let $G$ be a finite group. Then

$$
\begin{aligned}
& G \simeq A_{5} \text { iff } \pi_{e}(G)=\{1,2,3,5\} \quad[8] ; \\
& G \simeq L_{2}(7) \text { iff } \pi_{e}(G)=\{1,2,3,4,7\} \quad[6] ; \\
& G \simeq L_{2}(8) \text { iff } \pi_{e}(G)=\{1,2,3,7,9\} \quad[4] ; \\
& G \simeq L_{2}(17) \text { iff } \pi_{e}(G)=\{1,2,3,4,8,9,17\} \quad[1] ; \\
& G \simeq L_{3}(4) \text { iff } \pi_{e}(G)=\{1,2,3,4,5,7\} \quad[7] ; \\
& G \simeq S_{z}(8) \text { iff } \pi_{e}(G)=\{1,2,4,5,7,13\} ; \\
& G \simeq S_{z}(32) \text { iff } \pi_{e}(G)=\{1,2,4,5,25,31,41\}
\end{aligned}
$$

Open problem. How many finite simple groups $G$ can be characterized using only the condition of $\pi_{e}(G)$ ? I think it is a difficulty problem.

\section{ACKNOWLEDGMENT}

The author is grateful to the referee for improving the proof.

\section{REFERENCES}

1. R. Brandl, Finite groups all of whose elements are of prime power order, Boll. Un. Mat. Ital. A. (6) 18 (1981), 491-493.

2. G. Higman, Finite groups in which every element has prime power order, J. London Math. Soc. 32 (1957), 335-342.

3. B. Huppert and N. Blackburn, Finite Groups III, Springer-Verlag, Berlin, Heidelberg, and New York, 1982.

4. Shi Wujie, $A$ characteristic property of $J_{1}$ and $\mathrm{PSL}_{2}\left(2^{n}\right)$, Adv. in Math. 16 (1987), 397401. (Chinese)

5. __, A new characterization of some simple groups of Lie type, Contemp. Math. 82 (1989), 171-180.

6. __ A characteristic property of $\mathrm{PSL}_{2}$ (7), J. Austral. Math. Soc. Ser. A 36 (1984), 354356.

7. _ A characterization of some projective special linear groups, J. of Math. (PRC) 5 (1985), 191-200.

8. (1986), 11-14. (Chinese)

9. M. Suzuki, A new type of simple groups of finite order, Proc. Nat. Acad. Sci. U. S. A. 46 (1960), 868-870.

10. _ Finite groups with nilpotent centralizers, Trans. Amer. Math. Soc. 99 (1961), 425-470.

11. _ On a class of doubly transitive groups, Ann. Math. 75 (1962), 105-145.

Department of Pure Mathematics, The University of Sydney, N. S. W. 2006, Australia

Current address: Department of Mathematics, Southwest-China Normal University, Chongqing, 630715, People's Republic of China 